\title{
Exosomes from Human Gingiva-Derived Mesenchymal Stem Cells Combined with Biodegradable Chitin Conduits Promote Rat Sciatic Nerve Regeneration
}

\author{
Feng Rao $\mathbb{D}^{1},{ }^{1}$ Dianying Zhang, ${ }^{1}$ Tengjiaozi Fang, ${ }^{2}$ Changfeng Lu, ${ }^{1,3}$ Bo Wang, ${ }^{1}$ Xiao Ding, ${ }^{3}$ \\ Shuai Wei, ${ }^{3}$ Yiran Zhang, ${ }^{1}$ Wei Pi, ${ }^{1}$ Hailin Xu, ${ }^{1}$ Yanhua Wang, ${ }^{1}$ Baoguo Jiang ${ }^{D},{ }^{1}$ \\ and Peixun Zhang $\mathbb{D D}^{1}$ \\ ${ }^{1}$ Department of Orthopedics and Trauma, Peking University People's Hospital, Beijing, China \\ ${ }^{2}$ Department of Pediatric Dentistry, Peking University School and Hospital of Stomatology, National Engineering Laboratory for \\ Digital and Material Technology of Stomatology, and Beijing Key Laboratory of Digital Stomatology, Beijing 100081, China \\ ${ }^{3}$ Institute of Orthopedics, Chinese PLA General Hospital, Beijing Key Lab of Regenerative Medicine in Orthopedics, Beijing, China
}

Correspondence should be addressed to Baoguo Jiang; jiangbaoguo@vip.sina.com and Peixun Zhang; zhangpeixun@bjmu.edu.cn

Received 30 November 2018; Accepted 20 February 2019; Published 2 May 2019

Academic Editor: Alessandro Faroni

Copyright () 2019 Feng Rao et al. This is an open access article distributed under the Creative Commons Attribution License, which permits unrestricted use, distribution, and reproduction in any medium, provided the original work is properly cited.

\begin{abstract}
At present, repair methods for peripheral nerve injury often fail to get satisfactory result. Although various strategies have been adopted to investigate the microenvironment after peripheral nerve injury, the underlying molecular mechanisms of neurite outgrowth remain unclear. In this study, we evaluate the effects of exosomes from gingival mesenchymal stem cells (GMSCs) combined with biodegradable chitin conduits on peripheral nerve regeneration. GMSCs were isolated from human gingival tissue and characterized by surface antigen analysis and in vitro multipotent differentiation. The cell supernatant was collected to isolate the exosomes. The exosomes were characterized by transmission electron microscopy, Western blot, and size distribution analysis. The effects of exosomes on peripheral nerve regeneration in vitro were evaluated by coculture with Schwann cells and DRGs. The chitin conduit was prepared and combined with the exosomes to repair rat sciatic nerve defect. Histology, electrophysiology, and gait analysis were used to test the effects of exosomes on sciatic nerve function recovery in vivo. We have successfully cultured GMSCs and isolated exosomes. The exosomes from GMSCs could significantly promote Schwann cell proliferation and DRG axon growth. The in vivo studies showed that chitin conduit combined with exosomes from GMSCs could significantly increase the number and diameter of nerve fibers and promote myelin formation. In addition, muscle function, nerve conduction function, and motor function were also obviously recovered. In summary, this study suggests that GMSC-derived exosomes combined with biodegradable chitin conduits are a useful and novel therapeutic intervention in peripheral nerve repair.
\end{abstract}

\section{Introduction}

Every year, a large number of patients suffer from peripheral nerve injury worldwide, and the lack of effective treatments often leads to disability, which imposes a heavy burden on families and society [1-3]. There are many surgical methods to repair peripheral nerve injuries, and endto-end anastomosis is usually used for large injuries. Long peripheral nerve defects, from traumatic injuries, often require grafts to bridge the gap. Although autologous nerve transplantation remains the preferred strategy for reconstruction, it is limited by donor tissues, the sacrifice of functional nerves, and the potential formation of neuromas [4-6].

The repair of peripheral nerve injury is a very complicated pathological process, and it is difficult to obtain satisfactory results because of slow nerve regeneration, Wallerian degeneration, tissue adhesion, and muscle and motor endplate atrophy. Wallerian degeneration occurs after peripheral nerve injury, after which macrophages infiltrate the injured nerve and produce large amounts of inflammatory factors including CCL2, TNF-a, IL-1a, and IL-1beta $[7,8]$. 
The development of new therapeutic strategies to accelerate neurite outgrowth is of great importance [9]. Accordingly, mesenchymal stem cells (MSCs) have been used therapeutically for tissue regeneration and to treat autoimmune diseases $[10,11]$. Recent evidence suggests that a variety of mechanisms contribute to MSC-based therapies in which cytokines and growth factors can act as paracrine or autocrine mediators to modulate immune responses and tissue regeneration $[12,13]$.

Exosomes are extracellular vesicles that are secreted by living cells. They are released into the extracellular space through fusion of polyvesicular bodies and cytoplasmic membranes. The diameter of exosomes ranges from 30 to $150 \mathrm{~nm}$, and their size is similar to that of viruses [14]. Further, exosomes are widely involved in intercellular communication and play an important role in tissue repair and regeneration, immune regulation, and organism development $[15,16]$. Stem cell-derived exosomes can repair skin defects [17], treat fractures [18], promote cartilage defect repair [19], regulate microglial M1/M2 polarization, enhance brain injury repair [20], and facilitate the outward growth of neural axons after trauma [21].

Vasculogenesis plays an important physiological role in tissue repair. Specifically, it can result in the transport of oxygen, nutrients, and immune cells to the injured site. Moreover, many studies have found that cytogenic exosomes can participate in signaling pathways involved in angiogenesis, affecting the development and maturation of blood vessels [22].

Immunoinflammation plays an important role in coordinating a series of physiological processes involved in tissue repair and regeneration. However, it remains a challenge to control inflammation. Many studies have confirmed that MSC-derived exosomes can mimic the ability of MSCs to regulate immune cells including $B$ cells, T cells, natural killer cells, dendritic cells, and macrophages [23, 24]. Gingival mesenchymal stem cells (GMSCs) are stem cell-specific precursor cells that can be isolated from gingival tissues [25-27] and have the capacity for self-renewal, multidirectional differentiation, and immune regulation. GMSCs present different advantages, including their collection which did not require invasive procedures and allow MSC collection from the patients for autologous transplantation. Moreover, they had different advantages compared to bone marrow-derived MSCs. Indeed, GMSCs are easy to isolate, homogenous, and proliferate faster than bone marrow-derived MSCs, present a stable morphology, and maintain a normal karyotype and MSC characteristics also after long-term cultures [28-30]. However, whether exosomes derived from gingival stem cells can promote peripheral nerve regeneration has not been reported. In this study, we used a biodegradable chitin conduit combined with gingival stem cell-derived exosomes to bridge a $10 \mathrm{~mm}$ sciatic nerve defect in rats to address this question.

\section{Materials and Methods}

2.1. Culture of Human Gingiva-Derived Mesenchymal Stem Cells. With the approval of the ethical committee of the Peking University Hospital of Stomatology, gingivae were collected from four healthy patients without a history of periodontal disease, such as upon wisdom tooth extraction. Human GMSCs were isolated as described in published protocols [31]. The gingival tissues were treated aseptically, washed several times with PBS, and cultured in a medium containing $2 \mathrm{mg} / \mathrm{mL}$ protease (Gibco) at $4^{\circ} \mathrm{C}$. After terminating the digestion, the epithelial layer and the lamina propria were separated, and the lamina propria was collected and shredded. Tissues were cut into pieces and digested with collagenase IV at $37^{\circ} \mathrm{C}$ for 1 hour and centrifuged at $1000 \mathrm{rpm}$ for 10 minutes, and the supernatant was discarded. The cells were suspended in a $10 \mathrm{~cm}$ cell culture dish and cultured in a humidified cell culture incubator containing $5 \% \mathrm{CO}_{2}$ at $37^{\circ} \mathrm{C}$ for 24 hours in alpha-MEM medium containing 10\% FBS and $100 \mathrm{U} / \mathrm{mL}$ penicillin $/ 100 \mu \mathrm{g} / \mathrm{mL}$ streptomycin. The original culture medium was discarded, and the floating cells were removed. Fresh medium was added. The medium was then replaced every two days. When cell fusion reached $80 \%$, cells were digested using trypsin for continuous passage. Passages from generations 3-5 were used for experiments.

2.2. Flow Cytometric Analysis. Cells $\left(2 \times 10^{5}\right)$ were incubated with a specific monoclonal antibody conjugated with either fluorescein isothiocyanate (FITC) or phycoerythrin (PE) in $200 \mu \mathrm{L}$ PBS (SH30256.01; HyClone) for $30 \mathrm{~min}$ in the dark at $4^{\circ} \mathrm{C}$. The cell surface antigens were then analyzed by flow cytometry. Antibodies against CD34 (PE) (ab187284; Abcam), CD45 (PE) (ab134202; Abcam), CD73 (FITC) (ab239246; Abcam), CD90 (FITC) (ab124527; Abcam), and CD146 (FITC) (ab78451; Abcam) were used. Mouse monoclonal IgG1 (ab170190; Abcam) isotype was used as control.

\subsection{Multipotent Differentiation of GMSCs}

2.3.1. Osteogenic Differentiation. GMSCs were plated at $5 \times 10^{5}$ cells/well in 6-well plates in MSC growth medium; upon reaching $60 \%$ fusion, medium was replaced with osteogenic induction medium supplemented with dexamethasone, L-glutamine, ascorbic acid, and $\beta$-glycerophosphate. The culture medium was changed every three days. After 4 weeks, in vitro mineralization was assayed by Alizarin Red S staining.

2.3.2. Adipogenic Differentiation. When GMSCs reached $60 \%$ fusion, the medium was replaced with adipogenic induction culture medium consisting of MEM medium with $10 \mu \mathrm{M}$ human insulin, $1 \mu \mathrm{M}$ dexamethasone, $200 \mu \mathrm{M}$ indomethacin, and $0.5 \mathrm{mM} 3$-isobutyl-1-methylxanthine. The medium was replaced every three days for 14 days. Oil Red O staining was performed to detect intracellular lipid vacuole characteristic of adipocytes.

2.4. Culture and Proliferation Assay of the Schwann Cell. Three-day-old Sprague-Dawley (SD) mice were used. The bilateral sciatic nerves were cut, and its epicardium was removed. Sciatic nerve was collected and digested with $0.2 \%$ NB4 collagenase for $10 \mathrm{~min}$ at $37^{\circ} \mathrm{C}$. Schwann cell culture medium (10\% fetal bovine serum, $1 \%$ penicillin/streptomycin, and $10^{-2} \mathrm{M} / \mathrm{mL}$ forskolin) was added to resuspend the 
cells. Schwann cell was seeded in a cell culture flask. After $48 \mathrm{~h}$, Schwann cell was digested and seeded in 96-well plates and 6-well plates cultured with exosomes $(100 \mu \mathrm{g} / \mathrm{mL})$. After culture for 1,3 , and 5 days, $10 \mu \mathrm{L}$ of CCK solution was added to $96-$-well plates. The absorbance at $450 \mathrm{~nm}$ was measured with a microplate reader. Five days after culture, Schwann cell in 6-well plates was identified by immunofluorescence staining using rabbit anti-S100 antibody.

2.5. Isolation and Identification of Exosomes. GMSCs were cultured at $3 \times 10^{6}$ cells $/ 75 \mathrm{~cm}^{2}$ density with $15 \mathrm{~mL}$ medium containing exosomes-free fetal bovine serum for $48-72 \mathrm{~h}$; then, the medium was collected in a $15 \mathrm{~mL}$ centrifugal tube and centrifuged at $3000 \times \mathrm{g}$ for $10 \mathrm{~min}$; the supernatant was then carefully collected and transferred to a new tube and placed on ice. The supernatant from $10 \mathrm{~mL}$ of cells was extracted, and $2.5 \mathrm{~mL}$ of the exosome extraction reagent (HieffTM Quick exosome isolation kit, 41201ES25) was added. The supernatant was mixed via vortex oscillation for 1 minute, and then placed at $4^{\circ} \mathrm{C}$ for $2 \mathrm{~h}$. The tube containing the mixture was removed and centrifuged for 60 minutes at $4^{\circ} \mathrm{C}$ and $10000 \times g$; then, the supernatant was discarded, and the pellet was collected. The centrifugal sediment was beaten uniformly with $100 \mu \mathrm{L}$ of PBS for mixing and transferred to a new $1.5 \mathrm{~mL}$ centrifugal tube. The $1.5 \mathrm{~mL}$ centrifugal tube containing exosome was centrifuged at $4^{\circ} \mathrm{C}$ for $2 \mathrm{~min}$ at $12000 \times \mathrm{g}$. The precipitation was discarded, and the supernatant was retained.

The protein content of the exosomes was detected by the BCA Protein Assay Kit (Thermo). The morphology of the exosomes was analyzed by transmission electron microscopy (JEM-1400 Plus). The size of exosomes was measured by nanoparticle tracking analyses (ZetaView). The exosomal characteristic markers, CD9 and CD63, were analyzed by Western blot analysis.

2.6. Isolation and Culture of Dorsal Root Ganglion (DRG) Cells with Exosomes. SD mice born within 24 hours were sacrificed. Then, $75 \%$ alcohol was used to sterilize the backs of mice; vertebrae were exposed and removed and then placed in DMEM-F12 medium containing 10\% FBS. The vertebral body was cut centrally along the sagittal plane, and the DRG was extracted from the intervertebral foramen and placed in a new culture dish. The DRG epineurium was then peeled off and plated onto poly-lysine-coated glass coverslips in 6-well plates. The DRGs were cultured with exosomes $(100 \mu \mathrm{g} / \mathrm{mL})$ in MEM medium (Gibco) supplemented with B-27 (Gibco) and GlutaMAX (Gibco). The control had no exosomes.

2.7. Immunofluorescence. After culture for 5 days, immunofluorescence staining was performed to evaluate the effect of exosomes on DRGs. First, DRG samples were fixed with paraformaldehyde for 30 minutes and then blocked with $10 \%$ goat serum for 1 hour. Next, mouse anti-neurofilament 200 (N0142; Sigma) and rabbit antiS100 (S2644; Sigma) were applied as the primary antibodies for overnight incubation at $4^{\circ} \mathrm{C}$. Goat anti-mouse IgG (Alexa Fluor ${ }^{\circledR} 488 ;$ Abcam) and goat anti-rabbit (Alexa
Fluor ${ }^{\circledR}$ 594; Abcam) secondary antibodies were then incubated with the samples in the dark for 1 hour. Three randomly selected DRG samples from each group were used for statistical analysis. The three longest axons per quadrant were measured and used to calculate the mean length of each DRG axon using the Image-Pro Plus 6.0 image analysis software.

2.8. Animals and Surgical Procedures. Chitin conduits (diameter: $1.2 \mathrm{~mm}$ ) were patented by the Peking University and China Spinning and Weaving Institute, and these can retain structure for at least 6 weeks in vivo and undergo complete biodegradation (please refer to Chinese patent ZL01136314 for technical details). Eight-week-old SD rats were provided by Beijing Weitong Lihua Co. Ltd. We followed the guideline for ethical review of animal welfare of China (GB/T 358922018), and all experiments complied with the relevant regulations of the Medical Ethics Committee of Peking University People's Hospital (approval no. 2102000024). Twenty-four SD rats weighing 200-220 g were randomly divided into three groups, with eight rats in each, as follows: the hollow chitin conduit group, chitin conduit plus GMSC-derived exosome group, and autograft group. All rats were anesthetized with a sodium pentobarbital solution $(30 \mathrm{mg} / \mathrm{kg}$ body weight), and then, the right hind limb was prepared. The sciatic nerve was cut and exposed, and $10 \mu \mathrm{L}$ PBS was administered to the hollow chitin conduit group, retaining a $10 \mathrm{~mm}$ gap. The exosome group received $10 \mu \mathrm{L}$ PBS containing $10 \mu \mathrm{g}$ exosomes. Then, the muscle and skin were sutured. After waking, the rats were automatically fed food and drinking water and were housed with a 12-hour light/dark cycle and natural circulation.

2.9. Transmission Electron Microscopy (TEM) and Morphological Analyses. Twelve weeks after surgery, the regenerated nerve tissue samples were removed and fixed with $2.5 \%$ glutaraldehyde at $4^{\circ} \mathrm{C}$ for 2 hours. Samples were stained with $1 \%$ osmium acid, dehydrated with a gradient of acetone, embedded in Epon812 epoxy resin, and then cut into $700 \mathrm{~nm}$ thick semithin sections and $70 \mathrm{~nm}$ thick ultrathin sections. Uranyl acetate and lead citrate were used to stain the $70 \mathrm{~nm}$ thick ultrathin sections, which were observed by transmission electron microscopy (JEM-1400 Plus). Semithin sections were stained with $1 \%$ toluidine blue and observed by light microscopy. The number of regenerated axons was recorded based on five random images per animal. The diameter of myelinated axons and the thickness of the myelin sheath were measured using the Image-Pro Plus software. Five random axons were counted for each random sample of five random TEM images.

2.10. Muscle Weight and Muscle Fiber Remodeling. Twelve weeks after the operation, bilateral gastrocnemius muscles were removed from each group and fixed with muscle tissue fixative mainly containing formaldehyde, glacial acetic acid, and absolute ethanol (G1111; Servicebio) for 24 hours, immediately after weighing. Paraffin-embedded tissues were cut into $7 \mathrm{~mL}$ thick sections and subjected to Masson's trichrome staining. Five random fields were imaged for each 

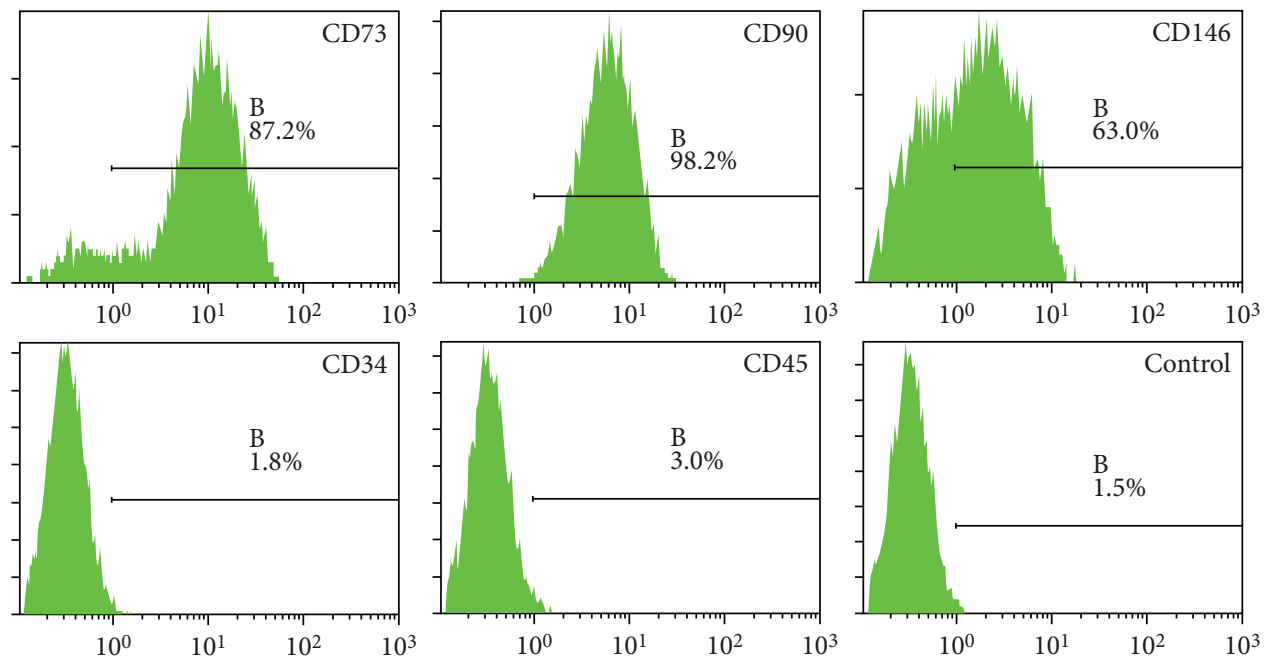

(a)
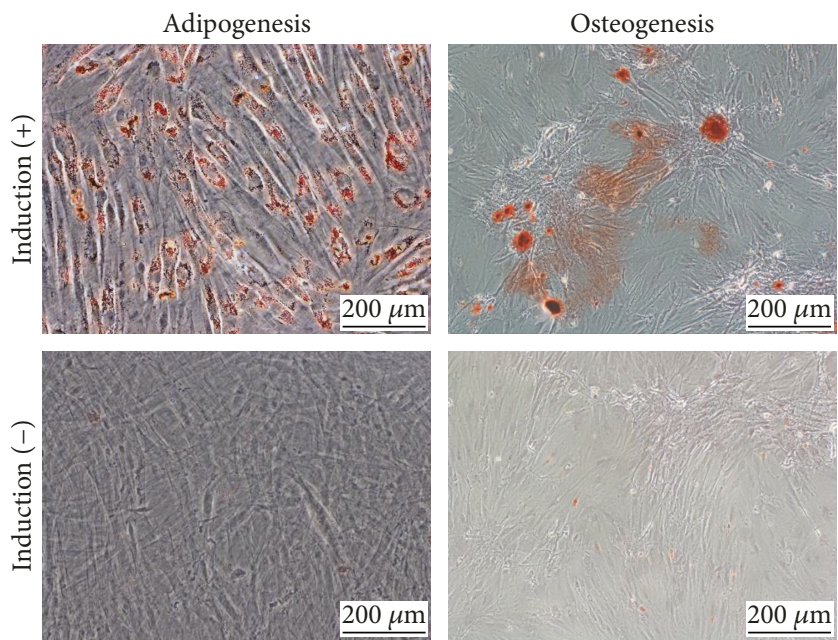

(b)

FIGURE 1: Characterization of GMSCs. (a) Flow cytometric analysis of surface markers of GMSCs. GMSCs were positive for MSC markers including CD73 (87.2\%), CD90 (98.2\%), and CD146 (63\%) and were negative for hematopoietic stem cell markers, CD34 (1.8\%) and CD45 (3.0\%). (b) Representative images of adipogenic differentiation and osteogenic differentiation. The visual field was filled with red lipid droplets stained with Oil Red $\mathrm{O}$ solution in images of adipogenic differentiation. Calcium nodules with burrs were formed in images of osteogenic differentiation, which were stained red by Alizarin Red solution.

sample by optical microscopy and quantitatively analyzed using Image-Pro Plus.

2.11. CatWalk Gait Analysis. The CatWalk XT 9.0 gait analysis system (Noldus, Wageningen, The Netherlands) was used to evaluate rat motor function recovery at 4,8 , and 12 weeks after surgery. The sciatic functional index (SFI) is a quantitative measure of sciatic nerve function. The formula is as follows: $\mathrm{SFI}=109.5(\mathrm{ETS}-\mathrm{NTS}) / \mathrm{NTS}-38.3(\mathrm{EPL}-$ $\mathrm{NPL}) / \mathrm{NPL}+13.3(\mathrm{EIT}-\mathrm{NIT}) / \mathrm{NIT}-8.8$.

2.12. Electrophysiological Assessment. At 12 weeks after surgery, the regenerated sciatic nerves were exposed, while mice were under anesthesia. Parameters of the Medlec Synergy electrophysiological system (Oxford Instrument Inc., Abingdon, UK) were set at a stimulus intensity of $0.09 \mathrm{~mA}$ and a duration of $0.1 \mathrm{~ms}$. The stimulating electrode was placed proximal to the sciatic nerve; the recording electrode was placed proximal and distal to the gastrocnemius muscle, and the reference electrode was placed on the gluteus maximus. Then, the CMAP latency and peak amplitude were calculated separately.

2.13. Statistical Analysis. All numerical data are expressed as the mean \pm standard deviation. Statistical analysis was performed by calculating the analysis of variance (ANOVA) followed by Tukey's post hoc multiple comparison test. All data were analyzed using SPSS 17.0. Differences were considered statistically significant with a $p$ value $<0.05$.

\section{Results}

3.1. Identification of Human Gingiva-Derived Mesenchymal Stem Cells. Flow cytometry analysis showed that GMSCs 


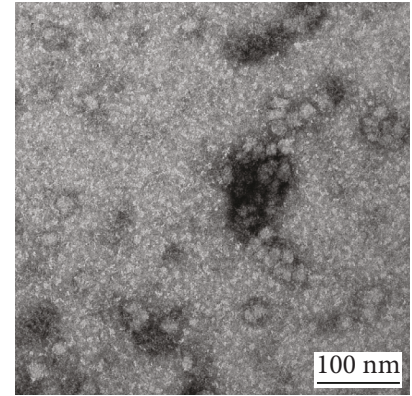

(a)

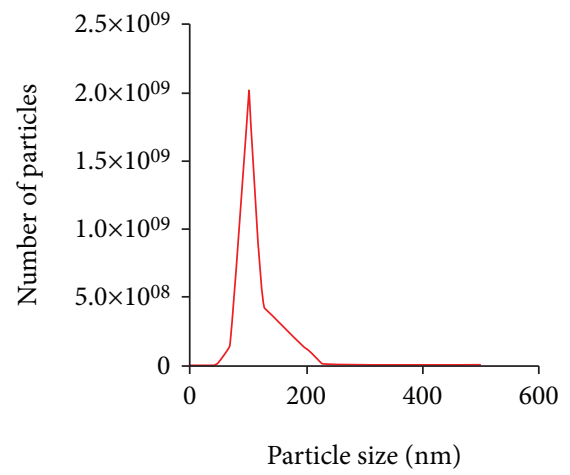

(b)

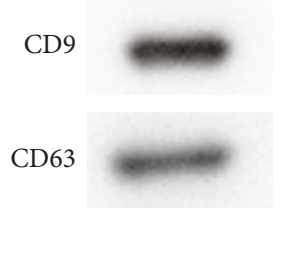

(c)

FIgURE 2: Characterization of exosomes from GMSC. (a) Representative images of exosomes from GMSC by TEM. (b) The size distribution profile of GMSC-derived exosomes. (c) Detection of exosomal markers (CD9 and CD63) by Western blot analysis.

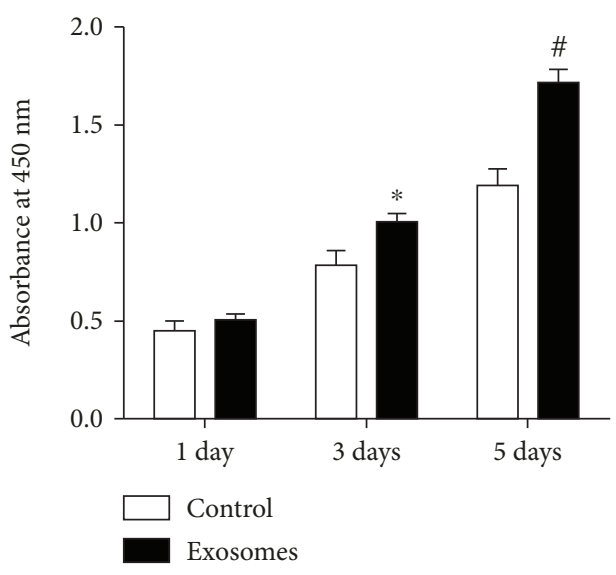

(a)
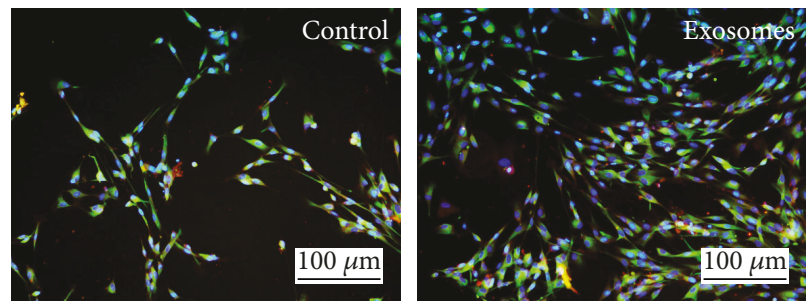

(b)

FIgURE 3: Exosomes promote Schwann cell proliferation. (a) Detection of the SC proliferation with CCK8. (b) S100 immunofluorescence of Schwann cells cultured with or without exosomes after 5 days. Results from 3 independent experiments with duplicates. Data are expressed as means \pm SEM. Statistical significance was obtained with one-way ANOVA with Tukey's post hoc test. ${ }^{*} p<0.05,{ }^{\#} p<0.01$.

were positive for MSC markers including CD73 (87.2\%), CD90 (98.2\%), and CD146 (63\%) and were negative for hematopoietic stem cell markers, CD34 (1.8\%) and CD45 (3.0\%). Also, CD106 is a marker of mesenchymal stem cells with strong immunoregulatory function (Figure 1(a)). Results of adipogenic and chondrogenic differentiation experiments demonstrated the differentiative potential of the GMSCs (Figure 1(b)).

3.2. Characterization of GMSC-Derived Exosomes. The ultrastructure of objects was observed by TEM. As shown in Figure 2(a), the exosomes exhibited a circular structure by TEM. Further, the size distribution of the purified exosomes was measured with the nanoparticle tracking system, which showed that the peak diameter was $102 \mathrm{~nm}$ (Figure 2(b)). Finally, the results of Western blot analysis revealed that GMSC-derived exosomes expressed the exosome markers CD9 and CD63 (Figure 2(c)).
3.3. Effects of GMSC-Derived Exosomes on SCs and DRG. Upon assessing the effect of GMSC-derived exosomes on SC outgrowth, CCK8 results showed no statistical difference between the two groups on the first day. On day 3, cell growth in the GMSC-derived exosome group was higher than that in the control group, and a statistical difference was noted between the groups $(p<0.05)$. On day 5, GMSC-derived exosomes were found to promote Schwann cell proliferation, and a statistical difference was noted between the two groups $(p<0.01)$ (Figure 3(a)). Immunofluorescence was also performed to show the Schwann cells of GMSC-derived exosomes and those of the control group on day 5 (Figure 3(b)). Also, immunofluorescence staining was performed to evaluate the effect of GMSC-derived exosomes on DRG neurite after culture for 5 days (Figure 4(a)). The length of DRG neurite cultured with GMSC-derived exosomes was significantly increased compared to the control $(p<0.01)$ (Figure 4(b)). 

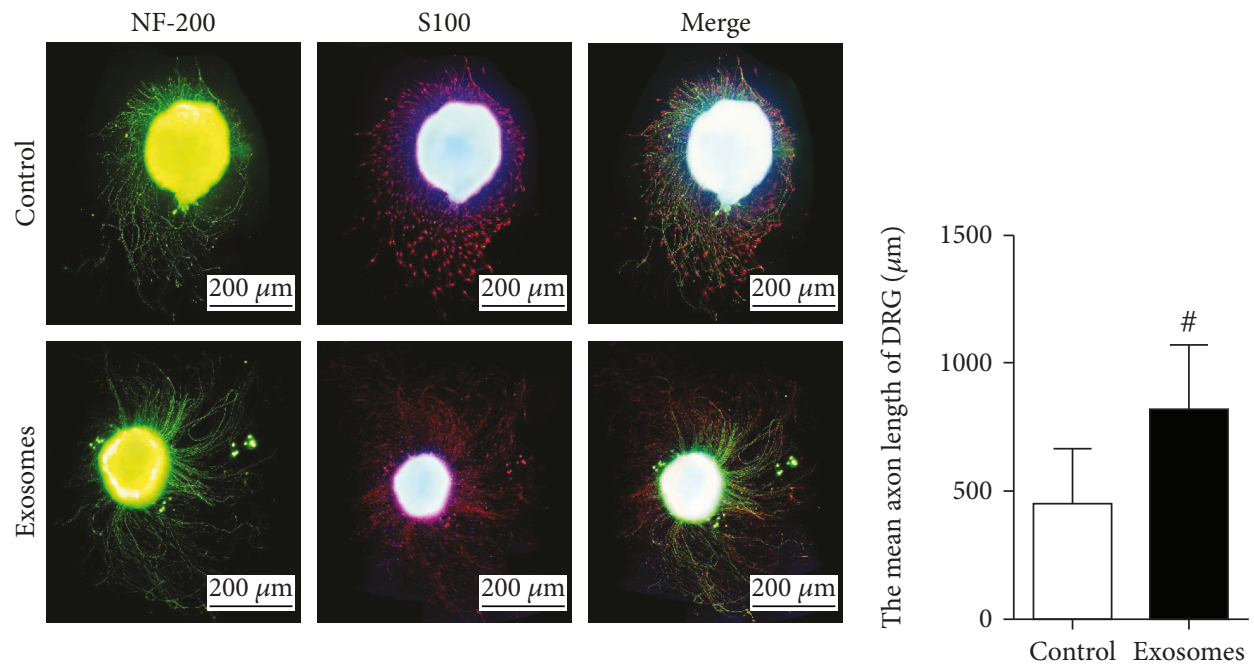

(a)

(b)

FIgURE 4: Exosomes enhance DRG neurite outgrowth. (a) DRGs were stained with NF200 (green) and S100 (red). (b) Statistical analysis of length of DRG neurite. Results from 4 independent experiments with duplicates. Data are expressed as means \pm SEM. Statistical significance was obtained with one-way ANOVA with Tukey's post hoc test, ${ }^{\#} p<0.01$.

3.4. Histological Evaluation of Regenerative Nerves. Toluidine blue staining of regenerated tissue revealed the formation of myelinated nerve fibers, and statistical analysis was performed to compare the numbers of regenerated nerve fibers (Figure 5(a)). The mean diameter and myelin sheath thickness of myelinated nerve fibers were quantified after TEM. This confirmed an increased number of regenerated nerve fibers in the GMSC-derived exosome group compared to the control group $(p<0.05)$ (Figure 5(b)); further, nerve fiber diameter and myelin sheath thickness were also improved with exosome administration $(p<0.05)$ (Figures 5(c) and 5(d)). However, there was still a gap, compared to that observed in the autograft group.

3.5. Histological Evaluation of the Gastrocnemius. The bilateral gastrocnemius muscles were isolated and assessed by Masson staining 12 weeks after the operation (Figure 6(a)). Cross-sectional images of muscles showed that crosssectional muscle fibers in the GMSC-derived exosome group were better than those in the control group $(p<0.05)$ (Figure 6(b)), and the muscle wet weight ratio was also improved compared to that in the control group $(p<0.05)$ (Figure 6(c)), which was consistent with SFI results.

3.6. Electrophysiological Evaluation. Electrophysiology was used to examine the electrical conduction of nerves (Figure $7(\mathrm{a})$ ). Electrical conduction is related to the number of myelinated nerve fibers and the thickness of the myelin sheath. The latency of CMAP is related to the thickness of the myelin sheath, whereas the CMAP amplitude is consistent with the number of nerve fibers. The latency in the GMSC-derived exosome group was significantly shorter, and the amplitude was significantly higher than that in the control group (both $p<0.01$ ) (Figure $7(\mathrm{~b})$ ).
3.7. Evaluation of Motor Function. Motor function evaluation was performed using a mouse gait analysis system, which can record paw prints in real time. Figure 8 (a) showed a threedimensional pressure diagram of paw prints. SFI was used to evaluate the recovery of motor function. At four-week postoperation, no statistical difference was found after comparing with the control group $(p>0.05)$ (Figure $8(b))$. Eight weeks after surgery, the SFI in the GMSC-derived exosome group was improved compared to that in the control group and this difference was statistically significant $(p<0.05)$ (Figure 8(b)). Twelve weeks after surgery, the SFI in the GMSC-derived exosome group was similar to that in the autograft group and was significantly improved compared to that in the hollow conduit group $(p<0.05)$ (Figure $8(b))$.

\section{Discussion}

Autologous nerve transplantation remains the gold standard for repairing peripheral nerve defects; however, it is necessary to uncover methods to replace this technique [32-34]. The local implantation of stem cells to treat peripheral nerve injury can promote axonal regeneration and myelin sheath formation. During the process of repair, stem cells secrete a variety of factors such as FGF, NGF, CNTF, BDNF, and GDNF, which can have a positive effect on neural cell survival and nerve regeneration [35-39]. Although stem cell-based therapies have shown beneficial effects on tissue regeneration, it has been reported that the main mechanism underlying stem cell-mediated tissue repair is paracrine rather than the differentiation of stem cells [40, 41]. Many studies have shown that the paracrine function of stem cells can be mediated by exosomes, which have significant potential to replace whole cell therapy as a new alternative method $[15,17,19,42]$. 

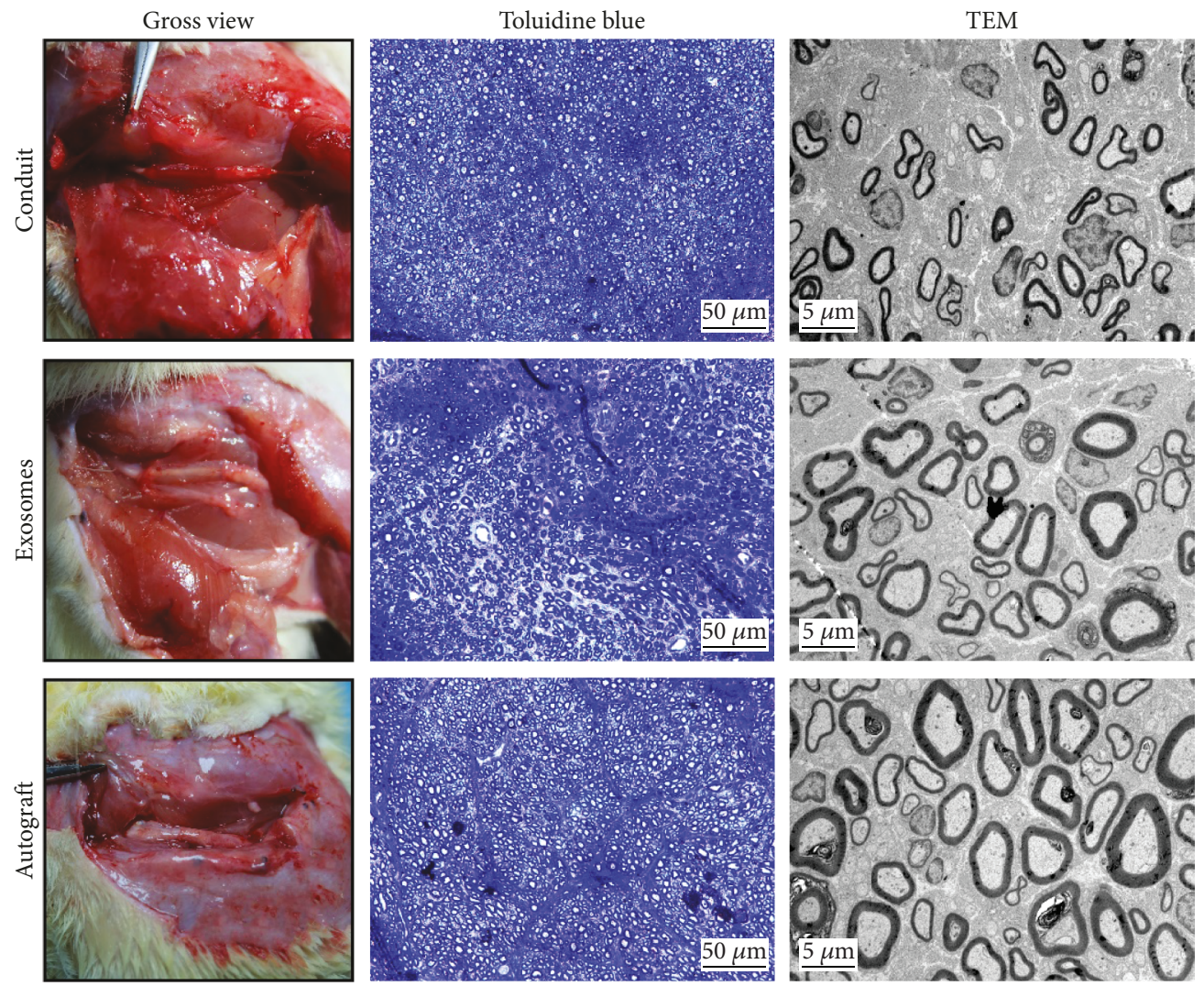

(a)

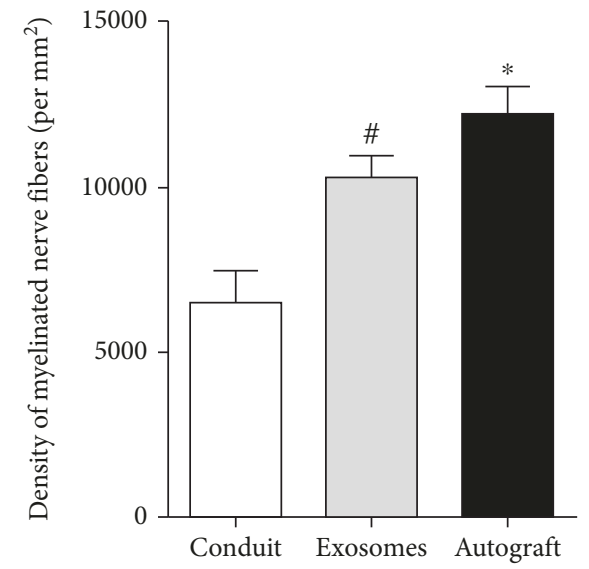

(b)

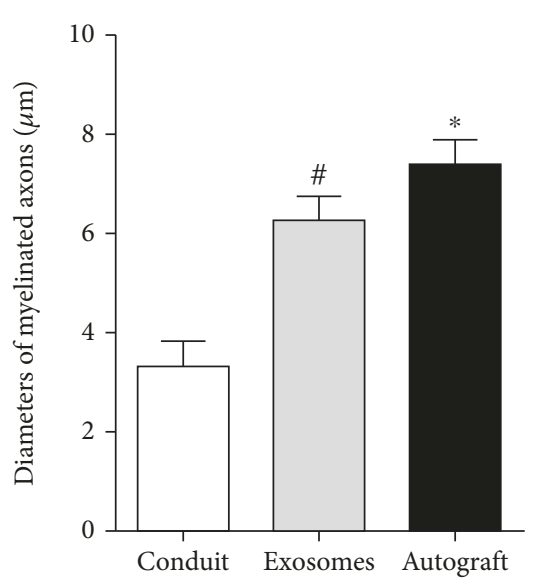

(c)

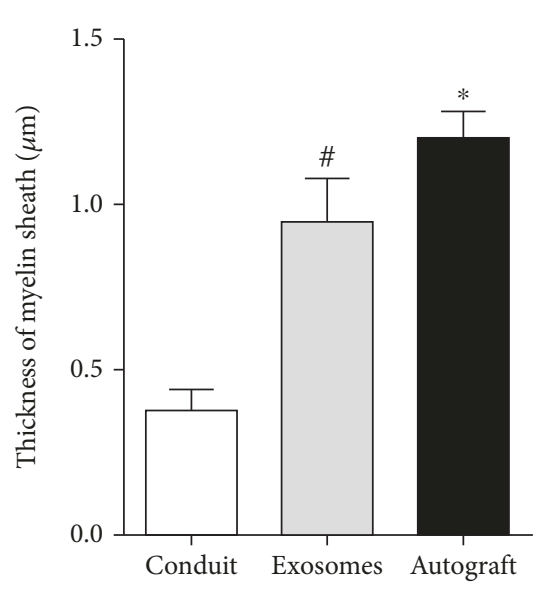

(d)

FIGURE 5: Evaluation of regenerated nerve fibers at 12 weeks after surgery. (a) Gross view, toluidine blue-stained transverse sections, and TEM images of the regenerated sciatic nerve. (b) Density of myelinated axons. (c) Diameters of myelinated axons. (d) Thickness of myelin sheath. Data are expressed as means \pm SEM $(n=8)$. Statistical significance was obtained with one-way ANOVA with Tukey's post hoc test. * $p<0.05$, ${ }^{\#} p<0.01$.

Stem cell-derived exosomes deliver various molecules including cytokines, growth factors, signaling lipids, mRNA, and microRNAs. Moreover, exosomes, as intercellular mediators of communication, transfer proteins, lipids, and RNA to regulate various physiological and pathological processes [43]. In addition, the application of exosomes was shown to be safer than stem cell administration, which could overcome cellular immune rejection and carcinogenic mutations [44].
Gingival tissue is known to heal very quickly after injury, and scarring is rare, due to the functions of GMSCs. These cells have many advantages, including the fact that they are homogeneous, nontumorigenic, easily separated, and phenotypically stable [45]. In the present study, we successfully isolated GMSCs from human gingival tissue, and these cells showed osteogenic and adipogenic differentiation capabilities. Moreover, the isolated GMSCs expressed specific surface antigens including CD73, CD90, and CD146, but did not 

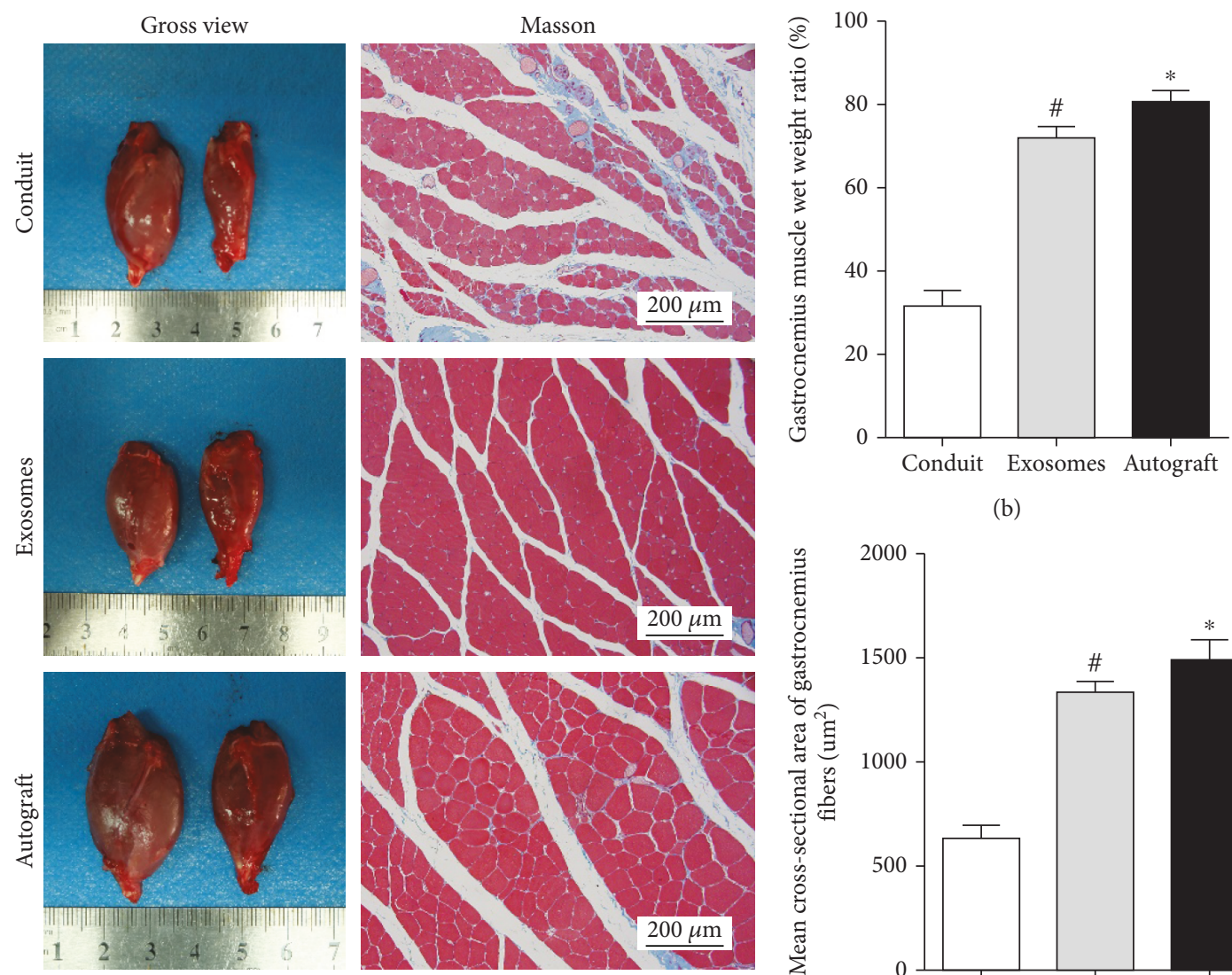

(b)

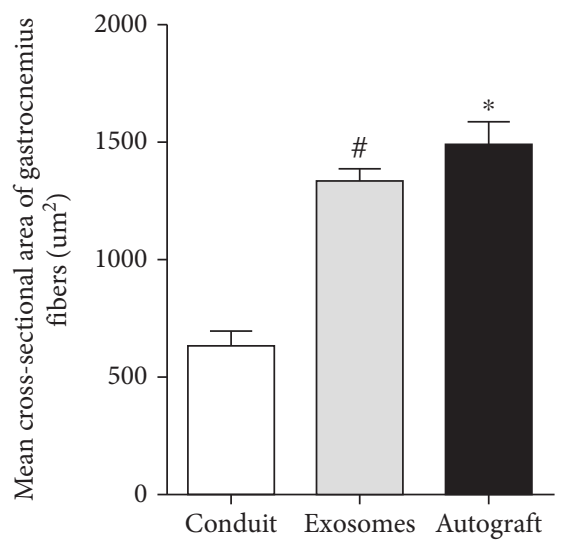

(a)

(c)

FIGURE 6: Reconstruction of muscle function at 12 weeks after surgery. (a) Gross images of gastrocnemius muscles and Masson's trichrome staining images. (b) Statistical analysis of the gastrocnemius muscle wet weight ratio. (c) Cross-sectional area of muscle fiber. Data are expressed as means $\pm \operatorname{SEM}(n=8)$. Statistical significance was obtained with one-way ANOVA with Tukey's post hoc test. ${ }^{*} p<0.05$, ${ }^{\#} p<0.01$.

express hematopoietic antigens such as CD34 and CD45. These results were consistent with the previous reports [31].

GMSC transplantation for peripheral nerve regeneration after injury has been applied in several studies [25, 27, 28, 38]. Lai et al. and Court et al. suggested that exosomes might modulate neurite outgrowth in the CNS $[46,47]$. However, to our knowledge, GMSC-derived exosomes have not been studied with respect to peripheral nerve defects. In our study, we also successfully isolated and characterized exosomes from GMSCs.

DRG culture in vitro is usually used to evaluate peripheral nerve regeneration. Our results showed for the first time that GMSC-derived exosomes can significantly promote DRG axonal growth. Lopez-Verrilli et al. found that exosomes derived from Schwann cells can significantly promote axonal growth in vitro and in vivo [48]. In the present study, we found that GMSC-derived exosomes could also significantly promote the proliferation of Schwann cells. Studies have reported that exosomes derived from adipose stem cells (ADMSCs) can be transferred into Schwann cells, which indicated that exosomes can be transferred between cells [49]. Also, exosomes of Schwann cell-like differentiated adipose stem cells express Gap43, Tau, Rac1, RhoA mRNAs,
miR-18a, miR-182, miR-21, miR-222, and miR-1miRNAs associated with neural regeneration [50].

Using chitin conduits, we have performed different studies on small gap sutures in vivo [51-53]. Typically, the effect of hollow conduits on the repair of $10 \mathrm{~mm}$ nerve defects is poor, and therefore, we used chitin conduits combined with exosomes derived from GMSCs to repair $10 \mathrm{~mm}$ peripheral nerve defects in rats.

Twelve weeks after the operation, GMSC-derived exosomes not only enhanced the density and diameter of regenerated axons but also increased the thickness of the regenerated myelin sheath, which was consistent with the results of in vitro studies.

At the same time, electrophysiology results showed that the CMAP amplitude in the exosome group was significantly higher than that in the control group, whereas the CMAP latency was decreased in the exosome group compared to the control group. Gait analysis was also used to evaluate the restoration of neuromuscular function in rats. Our study showed that at 8 and 12 weeks after the operation, the SFI was improved in the GMSC-derived exosome group compared to the control group. This was consistent with histological and electrophysiological data. However, regenerated 


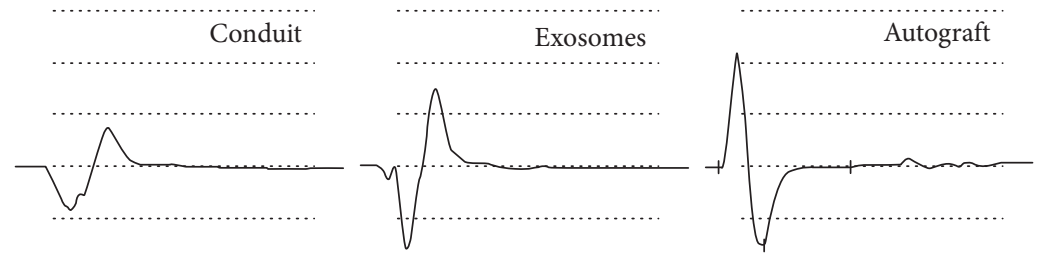

(a)

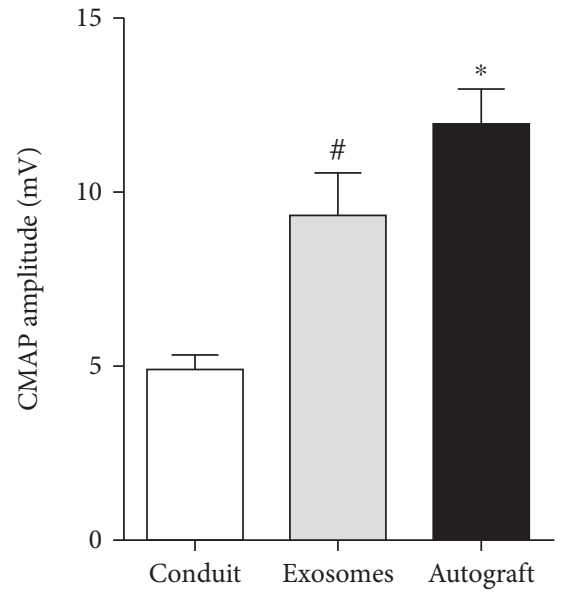

(b)

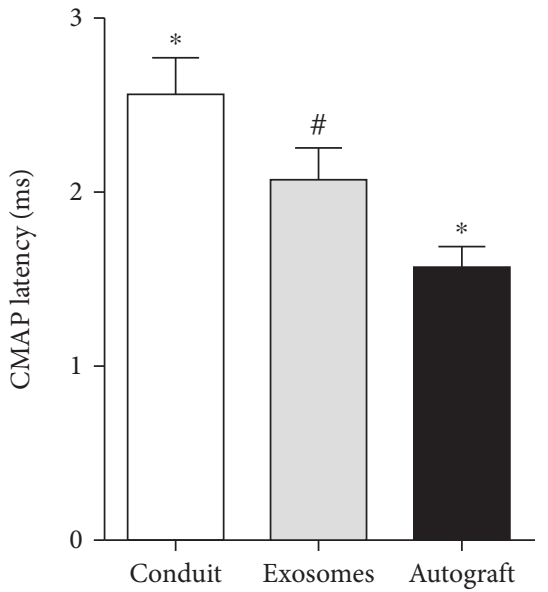

(c)

FIGURE 7: Electrophysiological detection of neuromuscular function at 12 weeks after surgery. (a) Representative CMAP recordings in each group. (b) Statistical analysis of CMAP amplitude and CMAP latency detected in each group. Data are expressed as means \pm SEM $(n=8)$. Statistical significance was obtained with one-way ANOVA with Tukey's post hoc test. ${ }^{*} p<0.05,{ }^{\#} p<0.01$.
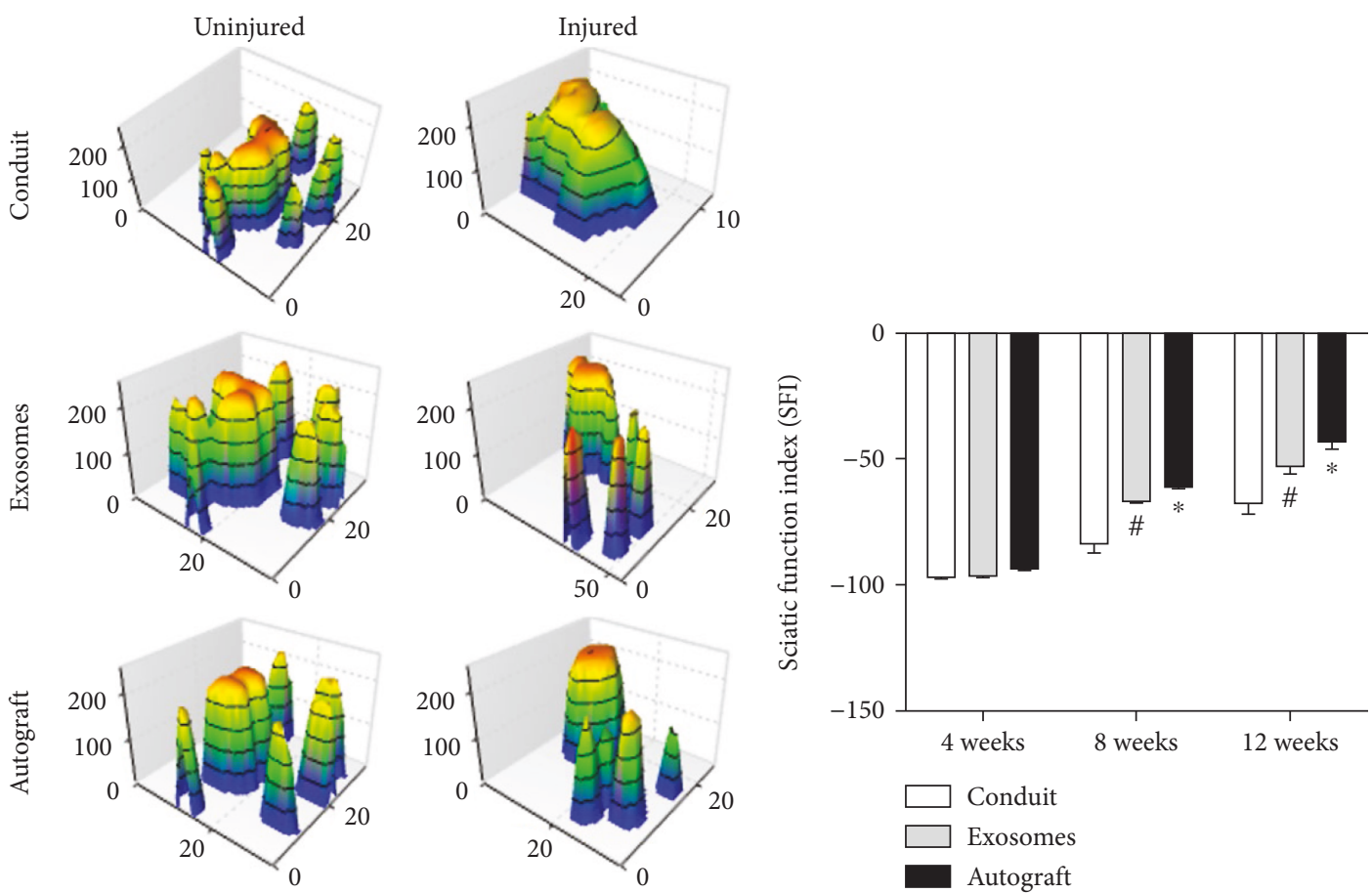

(a)

(b)

FIGURE 8: Motor functional recovery at 12 weeks by CatWalk gait analysis. (a) Representative 3D plantar pressure distribution of RH (injured) and LH (uninjured) of each group (by statistical analysis of SFI values). Data are expressed as means \pm SEM $(n=8)$. Statistical significance was obtained with one-way ANOVA with Tukey's post hoc test. ${ }^{*} p<0.05,{ }^{*} p<0.01$. 
nerve grows into the muscle until 4 weeks, and there was no statistical difference between the groups at this time.

In this study, 12 weeks after surgery, muscle histological data showed that the muscle wet weight and muscle fiber cross-sectional area were increased in the exosome group compared to the control group, indicating improved restoration of neuromuscular function, indirectly verifying the results of gait analysis.

Although GMSC-derived exosomes in chitin conduits have achieved good results in the treatment of peripheral nerve injury, the extraction method of exosomes needed further optimization to get more exosomes. Also, one remaining question was how gingival stem cell-derived exosomes function. Kou et al. found [54] that GMSC-derived exosomes have more protein content, including cytokines that can dampen the function of IL-1RA, which has an antiinflammatory effect. Coincidentally, IL-1RA is a known biological agent that can be used for the treatment of rheumatoid arthritis. Upon injection of IL-1RA into the wound, the wound healing rate was found to be greatly accelerated. In that study, the researchers found that GMSCs from diabetic mice secreted fewer exosomes than those from normal mice, resulting in a decrease in IL-1RA production. Moreover, the healing rate of diabetic mice was improved by applying IL-1RA from the GMSCs of healthy mice. Therefore, IL-1RA might play a crucial role in the repair of peripheral nerve injury. Our next study will examine whether IL-1RA promotes axonal growth and reduces scar formation.

\section{Conclusions}

In summary, the effect of exosomes on intercellular interactions is an exciting research area. Our results suggest that GMSC-derived exosomes combined with biodegradable chitin conduits represent a promising new approach for nerve regeneration.

\section{Data Availability}

All of the data and materials are available.

\section{Ethical Approval}

We followed the guideline for ethical review of animal welfare of China (GB/T 35892-2018), and all experiments complied with the relevant regulations of the Medical Ethics Committee of Peking University People's Hospital (approval no. 2102000024).

\section{Conflicts of Interest}

The authors declare no competing financial interests.

\section{Authors' Contributions}

F.R. and T.F. performed the majority of the experiments, analyzed the data, and prepared the manuscript. C.L., B.W., X.D., and S.W. assisted with rat in vivo experiments. Y.Z., W.P., and Y.W. helped with the in vitro experiments. B.J. and P.Z. supervised the project and wrote most of the manuscript. Feng Rao, Dianying Zhang, and Tengjiaozi Fang contributed equally to this work.

\section{Acknowledgments}

The authors would like to thank Kuan Chen from Beijing Hui Fukang Medical Device Co. Ltd. for his technical help about the preparation of chitin conduits and Xin Chang from School of Basic Medical Sciences, Peking University, for the help with transmission electron microscopy. This work was supported by the Major R\&D Program of National Ministry of Science and Technology (2018YFB1105504), National Key R\&D Program of China (2016YFC1101604), National Natural Science Foundation (31771322, 31571235, and 81671215), Beijing Municipal Science and Technology Commission Science and Technology Nova Cross Project (2018019), R\&D Foundation of PKUPH (2018-01, 201701), Ministry of Education Innovation Program of China (IRT_16R01), and National S\&T Major Project of China (SQ2018ZX100301).

\section{References}

[1] C. A. Taylor, D. Braza, J. B. Rice, and T. Dillingham, "The incidence of peripheral nerve injury in extremity trauma," American Journal of Physical Medicine \& Rehabilitation, vol. 87, no. 5, pp. 381-385, 2008.

[2] M. Asplund, M. Nilsson, A. Jacobsson, and H. von Holst, "Incidence of traumatic peripheral nerve injuries and amputations in Sweden between 1998 and 2006," Neuroepidemiology, vol. 32, no. 3, pp. 217-228, 2009.

[3] J. Noble, C. A. Munro, V. S. S. V. Prasad, and R. Midha, "Analysis of upper and lower extremity peripheral nerve injuries in a population of patients with multiple injuries," The Journal of Trauma, vol. 45, no. 1, pp. 116-122, 1998.

[4] W. Daly, L. Yao, D. Zeugolis, A. Windebank, and A. Pandit, “A biomaterials approach to peripheral nerve regeneration: bridging the peripheral nerve gap and enhancing functional recovery," Journal of the Royal Society, Interface, vol. 9, no. 67, pp. 202-221, 2012.

[5] Y. Gu, J. Zhu, C. Xue et al., "Chitosan/silk fibroin-based, Schwann cell-derived extracellular matrix-modified scaffolds for bridging rat sciatic nerve gaps," Biomaterials, vol. 35, no. 7, pp. 2253-2263, 2014.

[6] X. Hu, J. Huang, Z. Ye et al., "A novel scaffold with longitudinally oriented microchannels promotes peripheral nerve regeneration," Tissue Engineering Part A, vol. 15, no. 11, pp. 3297-3308, 2009.

[7] P. Chen, X. Piao, and P. Bonaldo, "Role of macrophages in Wallerian degeneration and axonal regeneration after peripheral nerve injury," Acta Neuropathologica, vol. 130, no. 5, pp. 605-618, 2015.

[8] A. DeFrancesco-Lisowitz, J. A. Lindborg, J. P. Niemi, and R. E. Zigmond, "The neuroimmunology of degeneration and regeneration in the peripheral nervous system," Neuroscience, vol. 302, pp. 174-203, 2015.

[9] X. Gu, F. Ding, and D. F. Williams, "Neural tissue engineering options for peripheral nerve regeneration," Biomaterials, vol. 35, no. 24, pp. 6143-6156, 2014. 
[10] Y. Liu, L. Wang, T. Kikuiri et al., "Mesenchymal stem cellbased tissue regeneration is governed by recipient $\mathrm{T}$ lymphocytes via IFN- $\gamma$ and TNF- $\alpha$," Nature Medicine, vol. 17, no. 12, pp. 1594-1601, 2011.

[11] K. Akiyama, C. Chen, D. Wang et al., "Mesenchymal-stemcell-induced immunoregulation involves FAS-ligand-/FASmediated T cell apoptosis," Cell Stem Cell, vol. 10, no. 5, pp. 544-555, 2012.

[12] M. E. Bernardo and W. E. Fibbe, "Mesenchymal stromal cells: sensors and switchers of inflammation," Cell Stem Cell, vol. 13, no. 4, pp. 392-402, 2013.

[13] D. G. Phinney, M. di Giuseppe, J. Njah et al., "Mesenchymal stem cells use extracellular vesicles to outsource mitophagy and shuttle microRNAs," Nature Communications, vol. 6, no. 1, p. 8472, 2015.

[14] C. Thery, L. Zitvogel, and S. Amigorena, "Exosomes: composition, biogenesis and function," Nature Reviews Immunology, vol. 2, no. 8, pp. 569-579, 2002.

[15] S. Kourembanas, "Exosomes: vehicles of intercellular signaling, biomarkers, and vectors of cell therapy," Annual Review of Physiology, vol. 77, no. 1, pp. 13-27, 2015.

[16] M. Tkach and C. Thery, "Communication by extracellular vesicles: where we are and where we need to go," Cell, vol. 164, no. 6, pp. 1226-1232, 2016.

[17] S. Rani and T. Ritter, "The exosome - a naturally secreted nanoparticle and its application to wound healing," Advanced Materials, vol. 28, no. 27, pp. 5542-5552, 2016.

[18] Z. C. Hao, J. Lu, S. Z. Wang, H. Wu, Y. T. Zhang, and S. G. Xu, "Stem cell-derived exosomes: a promising strategy for fracture healing," Cell Proliferation, vol. 50, no. 5, 2017.

[19] Y. Wang, D. Yu, Z. Liu et al., "Exosomes from embryonic mesenchymal stem cells alleviate osteoarthritis through balancing synthesis and degradation of cartilage extracellular matrix," Stem Cell Research \& Therapy, vol. 8, no. 1, p. 189, 2017.

[20] Y. Li, Y. Y. Yang, J. L. Ren, F. Xu, F. M. Chen, and A. Li, "Exosomes secreted by stem cells from human exfoliated deciduous teeth contribute to functional recovery after traumatic brain injury by shifting microglia M1/M2 polarization in rats," Stem Cell Research \& Therapy, vol. 8, no. 1, p. 198, 2017.

[21] S. Huang, X. Ge, J. Yu et al., "Increased mi R-124-3p in microglial exosomes following traumatic brain injury inhibits neuronal inflammation and contributes to neurite outgrowth via their transfer into neurons," The FASEB Journal, vol. 32, no. 1, pp. 512-528, 2018.

[22] D. Todorova, S. Simoncini, R. Lacroix, F. Sabatier, and F. Dignat-George, "Extracellular vesicles in angiogenesis," Circulation Research, vol. 120, no. 10, pp. 1658-1673, 2017.

[23] B. Zhang, Y. Yin, R. C. Lai, S. S. Tan, A. B. H. Choo, and S. K. Lim, "Mesenchymal stem cells secrete immunologically active exosomes," Stem Cells and Development, vol. 23, no. 11, pp. 1233-1244, 2014.

[24] J. Burrello, S. Monticone, C. Gai, Y. Gomez, S. Kholia, and G. Camussi, "Stem cell-derived extracellular vesicles and immune-modulation," Frontiers in Cell and Developmental Biology, vol. 4, no. 83, 2016.

[25] Q. Zhang, P. D. Nguyen, S. Shi et al., "Neural crest stem-like cells non-genetically induced from human gingiva-derived mesenchymal stem cells promote facial nerve regeneration in rats," Molecular Neurobiology, vol. 55, no. 8, pp. 6965-6983, 2018.
[26] Q. Zhang, S. Shi, Y. Liu et al., "Mesenchymal stem cells derived from human gingiva are capable of immunomodulatory functions and ameliorate inflammation-related tissue destruction in experimental colitis," Journal of Immunology, vol. 183, no. 12, pp. 7787-7798, 2009.

[27] S. Ansari, I. M. Diniz, C. Chen et al., "Human periodontal ligament- and gingiva-derived mesenchymal stem cells promote nerve regeneration when encapsulated in alginate/hyaluronic acid 3D scaffold," Advanced Healthcare Materials, vol. 6, no. 24, 2017.

[28] Q. Zhang, P. D. Nguyen, S. Shi, J. C. Burrell, D. K. Cullen, and A. D. Le, "3D bio-printed scaffold-free nerve constructs with human gingiva-derived mesenchymal stem cells promote rat facial nerve regeneration," Scientific Reports, vol. 8, no. 1, p. $6634,2018$.

[29] F. Diomede, N. Zini, J. Pizzicannella et al., "5-Aza exposure improves reprogramming process through embryoid body formation in human gingival stem cells," Front Genet, vol. 9, no. 419, 2018.

[30] F. Diomede, A. Gugliandolo, D. Scionti et al., "Biotherapeutic effect of gingival stem cells conditioned medium in bone tissue restoration," International Journal of Molecular Sciences, vol. 19, no. 2, 2018.

[31] Q. Shi, Z. Qian, D. Liu et al., "GMSC-derived exosomes combined with a chitosan/silk hydrogel sponge accelerates wound healing in a diabetic rat skin defect model," Frontiers in Physiology, vol. 8, no. 904, 2017.

[32] J. H. A. Bell and J. W. Haycock, "Next generation nerve guides: materials, fabrication, growth factors, and cell delivery," Tissue Engineering Part B, Reviews, vol. 18, no. 2, pp. 116-128, 2012.

[33] L. M. Marquardt and S. E. Sakiyama-Elbert, "Engineering peripheral nerve repair," Current Opinion in Biotechnology, vol. 24, no. 5, pp. 887-892, 2013.

[34] R. Li, Y. Li, Y. Wu et al., "Heparin-poloxamer thermosensitive hydrogel loaded with bFGF and NGF enhances peripheral nerve regeneration in diabetic rats," Biomaterials, vol. 168, pp. 24-37, 2018.

[35] M. H. Beigi, L. Ghasemi-Mobarakeh, M. P. Prabhakaran et al., "In vivo integration of poly( $\varepsilon$-caprolactone)/gelatin nanofibrous nerve guide seeded with teeth derived stem cells for peripheral nerve regeneration," Journal of Biomedical Materials Research Part A, vol. 102, no. 12, pp. 4554-4567, 2014.

[36] A. Del Fattore, R. Luciano, L. Pascucci et al., "Immunoregulatory effects of mesenchymal stem cell-derived extracellular vesicles on T lymphocytes," Cell Transplantation, vol. 24, no. 12, pp. 2615-2627, 2015.

[37] K. Sanen, W. Martens, M. Georgiou, M. Ameloot, I. Lambrichts, and J. Phillips, "Engineered neural tissue with Schwann cell differentiated human dental pulp stem cells: potential for peripheral nerve repair?," Journal of Tissue Engineering and Regenerative Medicine, vol. 11, no. 12, pp. 3362-3372, 2017.

[38] Q. Zhang, P. Nguyen, Q. Xu et al., "Neural progenitor-like cells induced from human gingiva-derived mesenchymal stem cells regulate myelination of Schwann cells in rat sciatic nerve regeneration," Stem Cells Translational Medicine, vol. 6, no. 2, pp. 458-470, 2017.

[39] J. Du, G. Zhen, H. Chen et al., "Optimal electrical stimulation boosts stem cell therapy in nerve regeneration," Biomaterials, vol. 181, pp. 347-359, 2018. 
[40] F. Togel, K. Weiss, Y. Yang, Z. Hu, P. Zhang, and C. Westenfelder, "Vasculotropic, paracrine actions of infused mesenchymal stem cells are important to the recovery from acute kidney injury," American Journal of Physiology-Renal Physiology, vol. 292, no. 5, pp. F1626-F1635, 2007.

[41] B. Zhang, X. Wu, X. Zhang et al., "Human umbilical cord mesenchymal stem cell exosomes enhance angiogenesis through the Wnt $4 / \beta$-catenin pathway," Stem Cells Translational Medicine, vol. 4, no. 5, pp. 513-522, 2015.

[42] D. G. Phinney and M. F. Pittenger, "Concise review: MSCderived exosomes for cell-free therapy," Stem Cells, vol. 35, no. 4, pp. 851-858, 2017.

[43] M. Yáñez-Mó, P. R. M. Siljander, Z. Andreu et al., "Biological properties of extracellular vesicles and their physiological functions," Journal of Extracellular Vesicles, vol. 4, no. 1, 2015.

[44] S. Rani, A. E. Ryan, M. D. Griffin, and T. Ritter, "Mesenchymal stem cell-derived extracellular vesicles: toward cell-free therapeutic applications," Molecular Therapy, vol. 23, no. 5, pp. 812-823, 2015.

[45] Y. Cao, X. Gang, C. Sun, and G. Wang, "Mesenchymal stem cells improve healing of diabetic foot ulcer," Journal of Diabetes Research, vol. 2017, Article ID 9328347, 10 pages, 2017.

[46] R. C. Lai, F. Arslan, M. M. Lee et al., "Exosome secreted by MSC reduces myocardial ischemia/reperfusion injury," Stem Cell Research, vol. 4, no. 3, pp. 214-222, 2010.

[47] F. A. Court, R. Midha, B. A. Cisterna et al., "Morphological evidence for a transport of ribosomes from Schwann cells to regenerating axons," Glia, vol. 59, no. 10, pp. 1529-1539, 2011.

[48] M. A. Lopez-Verrilli, F. Picou, and F. A. Court, "Schwann cellderived exosomes enhance axonal regeneration in the peripheral nervous system," Glia, vol. 61, no. 11, pp. 1795-1806, 2013.

[49] V. Bucan, D. Vaslaitis, C. T. Peck, S. Strauss, P. M. Vogt, and C. Radtke, "Effect of exosomes from rat adipose-derived mesenchymal stem cells on neurite outgrowth and sciatic nerve regeneration after crush injury," Molecular Neurobiology, vol. 56, no. 3, pp. 1812-1824, 2019.

[50] R. C. Ching, M. Wiberg, and P. J. Kingham, "Schwann cell-like differentiated adipose stem cells promote neurite outgrowth via secreted exosomes and RNA transfer," Stem Cell Research \& Therapy, vol. 9, no. 1, p. 266, 2018.

[51] B. Jiang, P. Zhang, and B. Jiang, "Advances in small gap sleeve bridging peripheral nerve injury," Artificial Cells, Blood Substitutes, and Immobilization Biotechnology, vol. 38, no. 1, pp. 1-4, 2010.

[52] B. Jiang, P. Zhang, D. Zhang, Z. Fu, X. Yin, and H. Zhang, "Study on small gap sleeve bridging peripheral nerve injury," Artificial Cells, Blood Substitutes, and Immobilization Biotechnology, vol. 34, no. 1, pp. 55-74, 2006.

[53] P. Zhang, X. Yin, Y. Kou et al., "Peripheral nerve mutilation through biodegradable conduit small gap tubulisation: a multicentre randomised trial," The Lancet, vol. 386, article S40, 2015.

[54] X. Kou, X. Xu, C. Chen et al., "The Fas/Fap-1/Cav-1 complex regulates IL-1RA secretion in mesenchymal stem cells to accelerate wound healing," Science Translational Medicine, vol. 10, no. 432, article eaai8524, 2018. 


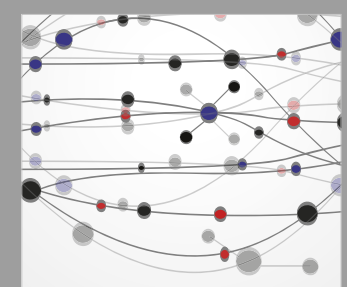

The Scientific World Journal
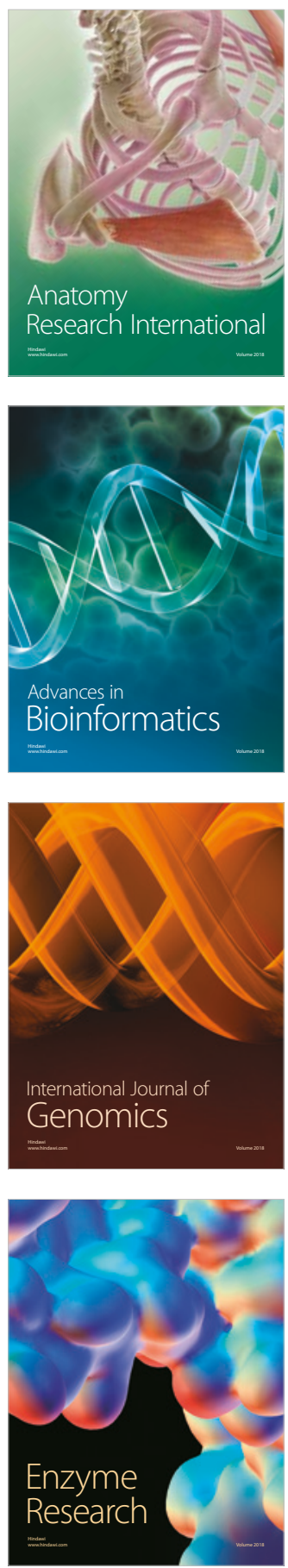
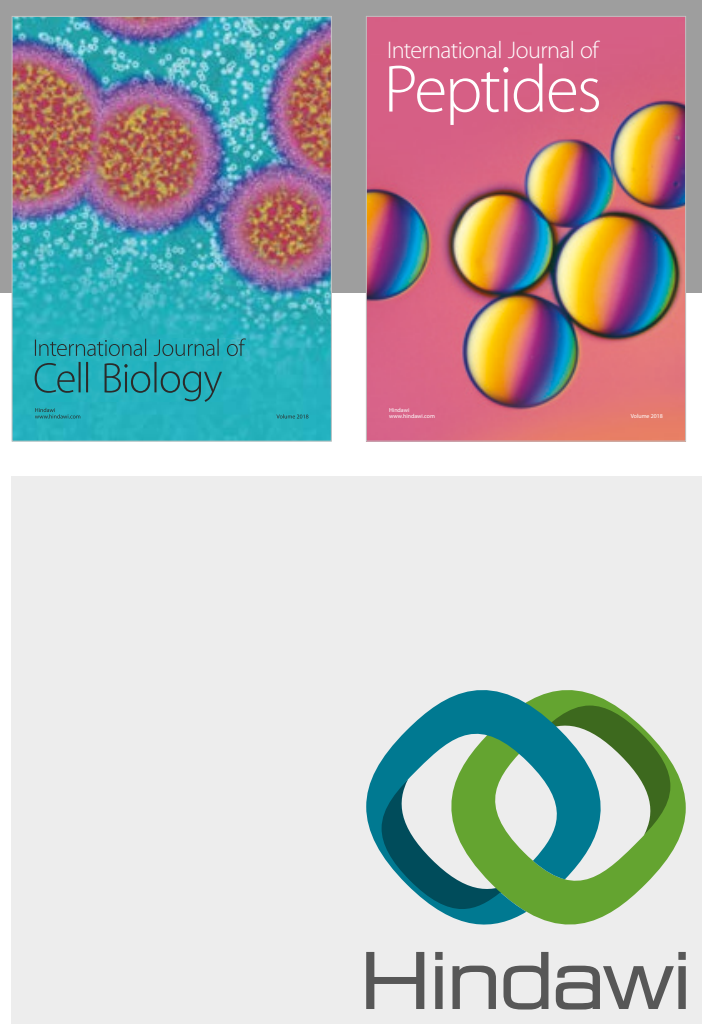

Submit your manuscripts at

www.hindawi.com
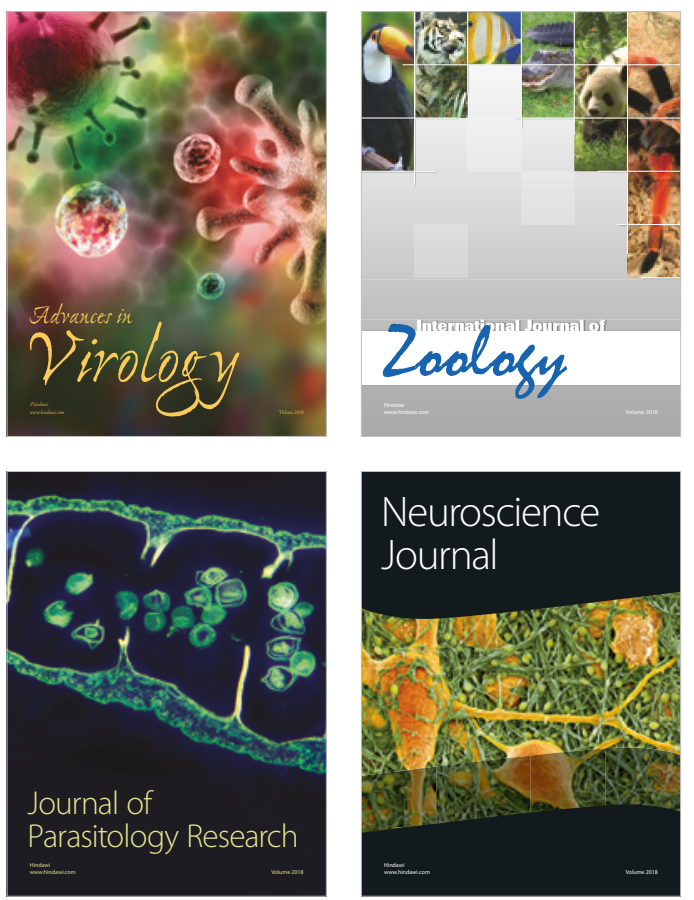
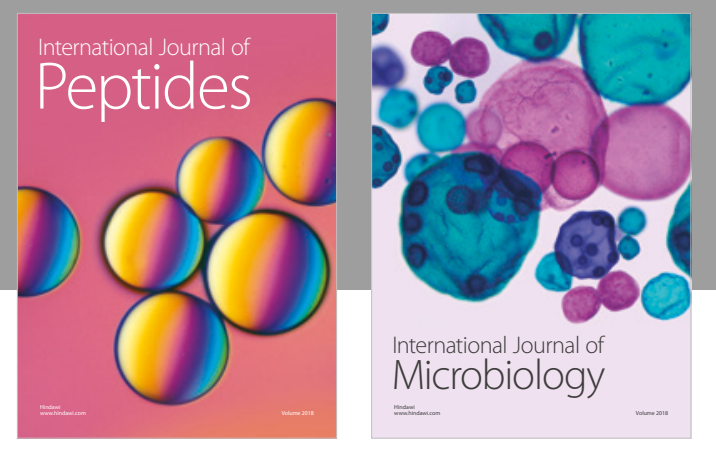

nternational Journal of Microbiology
Journal of
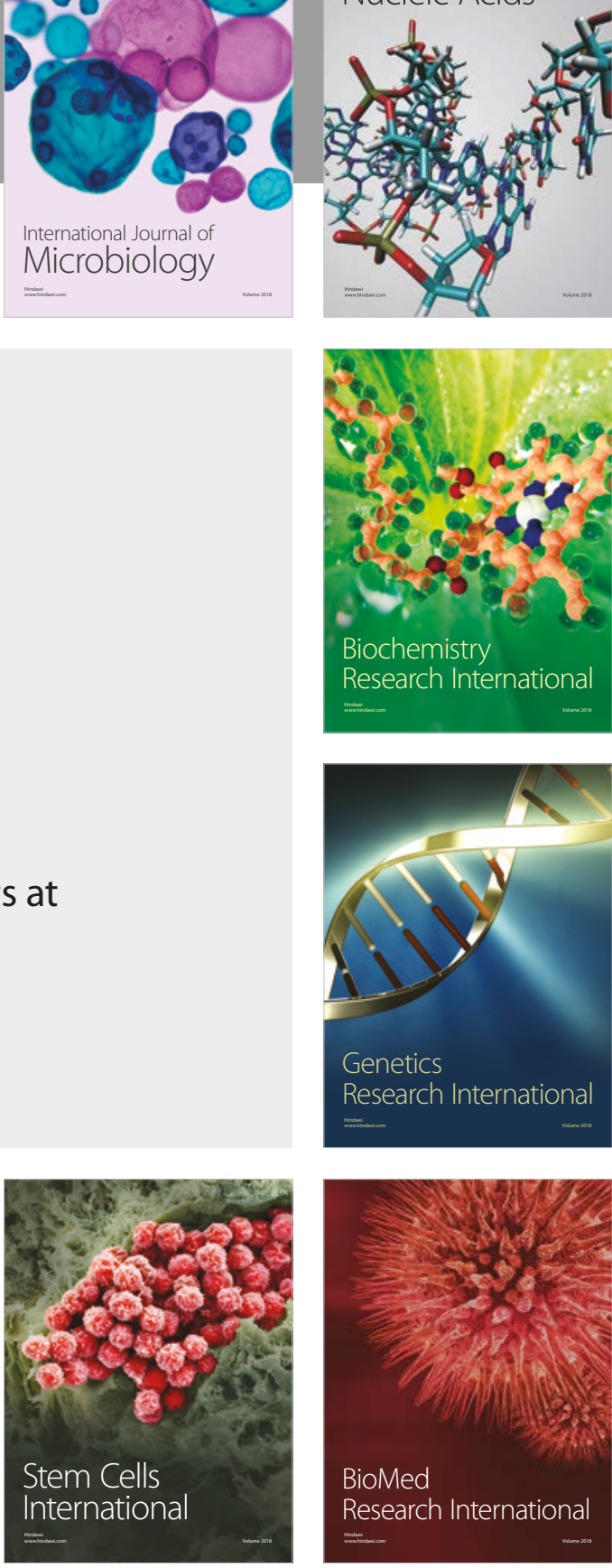
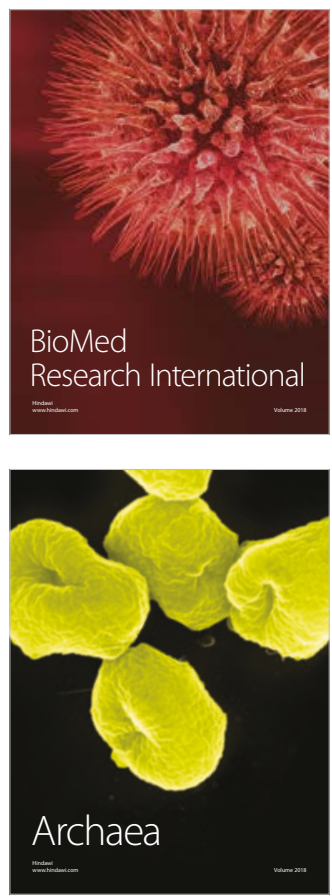